\title{
Amamentação natural, artificial e maloclusão: há correlação?
}

\author{
Breast-feeding, bottle-feeding and malocclusion: \\ is there correlation?
}

\section{RESUMO}

Introdução: o aleitamento materno é um fator essencial para o correto desenvolvimento do sistema estomatognático. Em geral, crianças que não recebem aleitamento materno apresentam tendência a maloclusão, porque dele a criança recebe os estímulos necessários para seu desenvolvimento craniofacial.

Objetivo: verificar a possível correlação entre tipo e tempo de aleitamento e desenvolvimento de maloclusões.

Método: realizou-se anamnese e exame clínico em 33 crianças com idade de 4 a 13 anos que buscaram atendimento na faculdade privada Universidade Metodista de São Paulo, em São Bernardo do Campo, SP. Os dados foram expressos em porcentagem e realizados ANOVA e teste de Tukey post hoc $(\mathrm{p}<0,05 \%)$.

Resultados: Foi observado que $69,7 \%$ das crianças receberam aleitamento artificial e $30,3 \%$ aleitamento natural $(p=0,001)$, sendo exclusivo ou não. $6,1 \%$ das crianças apresentavam dentadura decídua, $84,8 \%$ mista e $9,1 \%$ permanente. Nas dentaduras mista e permanente 54,8\% apresentavam Classe I, 35,5\% Classe II, e 9,7\% Classe III de Angle. Das crianças analisadas 40,0\% apresentavam sobressaliência e/ou sobremordida acentuada; $72,7 \%$ não apresentaram apinhamento contra $27,3 \%$ que apresentaram. Em $36,4 \%$ observou-se mordida cruzada, sendo que 50,0\% delas apresentava mordida cruzada posterior.

Conclusão: Não houve correlação entre maior chance de desenvolver maloclusão e maior tempo de aleitamento natural $(p=0,359)$ ou artificial $(p=0,244)$. O maior tempo de amamentação não indicará, necessariamente, maior tendência a maloclusão. Palavras chaves: Aleitamento materno; Má oclusão; Hábitos.

\section{ABSTRACT}

Introduction: breastfeeding is an essential factor for the correct development of the stomatognathic system. In general, children who don't receive breastfeeding are prone to malocclusion because from it the child receives the necessary incentive for their craniofacial development.

Objective: Correlate type and duration of breastfeeding to malocclusion.

Methods: anamnesis and clinical examination were carried out on 33 children aged 4 to 13 years who sought care at the private university Universidade Metodista de São Paulo, in São Bernardo do Campo, SP. Data were expressed as a percentage and ANOVA and Tukey post hoc test $(\mathrm{p}<0.05 \%)$ were performed.

Results: It was observed that $69.7 \%$ of the children received artificial breastfeeding and $30.3 \%$ natural breastfeeding $(\mathrm{p}=0.001)$, being exclusive or not. $6.1 \%$ of the children had deciduous teeth, $84.8 \%$ mixed and $9.1 \%$ permanent. In mixed and permanent dentures, 54.8\% had Class I, 35.5\% Class II, and 9.7\% Class III Angle. Of the children analyzed, $40.0 \%$ had overjet and / or severe overbite; $72.7 \%$ did not present crowding against $27.3 \%$ who did. In $36.4 \%$ a cross bite was observed, and $50.0 \%$ of them had a posterior cross bite.

Conclusion: There was no correlation between a greater chance of developing malocclusion and a longer duration of natural $(p=0.359)$ or artificial $(p=0.244)$ breastfeeding. The longer duration of breastfeeding does not indicate, however, a greater tendency to malocclusion.

Keywords: Breast feeding; Malocclusion; Habits.

\section{Agnes Martins Messias ${ }^{1}$ Sucena Matuk Long ${ }^{2}$ Maria Cristina Duarte Ferreira ${ }^{2}$ Érika Josgrillberg ${ }^{3}$ Renata Pilli Jóias ${ }^{3}$}

${ }^{1}$ DDS. Aluna do Curso de Especialização em Ortodontia, Universidade Metodista de São Paulo

${ }^{2}$ DDS, MsC, PhD. Professora do Curso de Odontologia, Universidade Metodista de São Paulo

3 DDS, MsC, PhD. Professora do Curso de Odontologia e do Curso de Especialização em Ortodontia, Universidade Metodista de São Paulo

Autor para correspondência:

Agnes Martins Messias

Rua Una, 41 - Parque João Ramalho - Santo André/SP. E-mail: agnesmartins_@hotmail.com; Tel (11) 974731259. 


\section{INTRODUÇÃO}

As estruturas integrantes do sistema estomatognático devem trabalhar em harmonia, sendo elas dentes, articulações, músculos, lábios, ligamentos, sem ocorrer alterações na mastigação, deglutição, fala e respiração. Quando ocorrem modificações no arranjo dos dentes, tonicidade dos músculos entre outras, podem ocorrer as maloclusões que impactam diretamente nas funções do sistema estomatognático. ${ }^{1}$

Essas funções devem ocorrer corretamente, pois são importantes para o desenvolvimento craniofacial, estimulando o equilíbrio durante o crescimento. Qualquer alteração nesse equilíbrio pode levar a uma anomalia estrutural dos ossos da face. ${ }^{2}$ Muitos fatores estão relacionados às maloclusões, como por exemplo hábitos bucais deletérios, traumatismos, fatores hereditários/congênitos, doenças sistêmicas/locais, agentes físicos, além dos fatores socioeconômicos e dieta pobre em nutrientes..$^{3-4}$ Os hábitos orais são classificados como deletérios e normais. A mastigação, deglutição e respiração nasal são hábitos fisiológicos e funcionais, pois são necessários para que uma oclusão normal seja estabelecida e assim contribuem para o desenvolvimento craniofacial. Os hábitos considerados deletérios são aqueles que não são fisiológicos, como chupeta, mamadeira, respiração bucal e sucção digital. ${ }^{5-6}$ Outros autores consideram sucção digital e chupeta como hábitos não nutritivos e mamadeira como sucção nutritiva. ${ }^{7-9}$

Em relação às medidas de prevenção de maloclusões, um dos fatores está na orientação as gestantes, em que se deve estimular à amamentar até no mínimo seis meses e também informar sobre alimentação saudável, visitas regulares ao dentista, boa higienização mecânica e a ocorrências dos hábitos orais, além de reforçar que a atenção pra saúde bucal deve ter início no primeiro ano de vida da criança, evitando assim alterações bucais. ${ }^{10-11}$

Uma vez que durante a gravidez o feto já pode desenvolver hábitos como sucção digital, interposição de língua e lábios, quando nasce, também já está apto a realizar sucção ao ser amamentado. Ao ser amamentada naturalmente, a criança suga o leite que a satisfaz trazendo um prazer oral, além de exercitar a musculatura e sentir-se segura. ${ }^{7}$ Em contrapartida, as crianças que são amamentadas por mamadeira, possuem tendência de colocar o dedo na boca para satisfazer a falta que a criança tem de sugar e sentir o prazer do alimento nutritivo vindo do seio materno. ${ }^{12}$

A amamentação artificial não necessita de esforços vindos do bebê e não satisfaz a necessidade de sucção e isso faz com que ela não desenvolva normalmente a musculatura e os ossos da face. Ao realizar uma amamentação precária, a criança vê necessidade de fazer sucção digital, dedo ou objetos e que se forem estendidos após os quatro anos de idade, leva a uma nocividade de reflexo sistêmico e gravidade variando de acordo com a intensidade, frequência e duração do hábito (Tríade de Graber) ${ }^{7}$. A Tríade de Graber, juntamente com a pressão empregada pelo dedo e às contrações bucais e labiais são fatores que devem ser considerados para observar alterações musculares, dentárias, ósseas e oclusais. ${ }^{13}$ Então, a criança cria hábitos deletérios que interferem diretamente na oclusão, pois nessa fase inicia-se o desenvolvimento da oclusão decídua. Quando esse desenvolvimento segue a normalidade, sem interferência de hábitos deletérios, favorece o crescimento facial, porém, a presença desses, pode originar desequilíbrios oclusais, gerando as maloclusões dentárias. 5,12 
A duração do aleitamento materno também influencia no surgimento das maloclusões, pois crianças que são amamentadas exclusivamente no seio por um período de no mínimo seis meses, de acordo com a OMS, possuem menor chance de desenvolver hábitos de sucção não nutritiva. ${ }^{14}$ Com o desmame precoce há maior chance da criança desenvolver hábitos orais deletérios como uso de chupeta, sucção digital, onicofagia, sucção de objetos, respiração bucal e bruxismo. ${ }^{15}$ As crianças que utilizaram dedo ou chupeta demonstraram risco 1,5 vezes maior (IC95\%=0,99 2,25) de maloclusão em comparação aquelas que não tiveram amamentação natural por um período de 4 meses. ${ }^{16}$ Em oposição outro estudo concluiu que as crianças que prolongaram o aleitamento materno até 24 meses (96,8\%; $\mathrm{n}=62$ ) desenvolveram algum hábito bucal. ${ }^{17}$

Por certo, os hábitos bucais deletérios podem desencadear desequilíbrios na musculatura facial, desenvolvendo assim maloclusões, porém isso vai depender de sua duração, frequência e intensidade. ${ }^{18} \mathrm{O}$ tipo de aleitamento e seu período de tempo, os hábitos deletérios e as maloclusões foram tema de muitos estudos, porém ainda há aspectos a serem melhor entendidos. Com base nisso este trabalho teve como objetivo observar a correlação entre tipo e duração da amamentação e desenvolvimento de maloclusões. Caso a hipótese nula $\left(H_{\mathrm{o}}\right)$ seja verdadeira, não haverá diferença estatisticamente significante entre o tipo e duração de amamentação e a tendência a maloclusão. Caso a hipótese alternativa $\left(H_{1}\right)$ seja verdadeira, o tipo ou duração da amamentação aumentará ou diminuirá a tendência a maloclusão.

\section{MATERIAIS E MÉTODOS}

O projeto de pesquisa desse trabalho foi aprovado pelo Comitê de Ética em Pesquisa com seres humanos (CAAE: 64480317.0.0000.5508), respeitando os princípios éticos e legais. Os voluntários da pesquisa foram informados por Termo de Consentimento Livre e Esclarecido.

\subsection{Amostra}

Foi obtida uma amostra de conveniência dentre os pacientes que buscaram atendimento na clínica infantil da faculdade de odontologia da Universidade Metodista de São Paulo, em São Bernardo do Campo, SP. A amostra foi constituída por 33 indivíduos, com idade entre 4 e 13 anos, 14 do sexo masculino e 19 do feminino.

\subsection{Métodos}

Foi realizada anamnese de cada indivíduo por meio de perguntas direcionadas aos pais/responsáveis. Obtiveram-se informações relacionadas à saúde geral da criança, idade, tipo de aleitamento (natural/artificial/misto) e presença de hábitos bucais de sucção não nutritivos (chupeta, digital) e sua duração por meio de anamnese dirigida aos pais ou responsáveis. Em seguida, foi realizado exame clínico supervisionado por um profissional calibrado para obtenção dos seguintes dados: classificação de Angle, tipo de dentição, desvio de linha média, mordida aberta, mordida cruzada, sobremordida, sobressaliência, apinhamento, cárie, perda precoce, fratura, anomalia de forma/tamanho, infraversão, vestibuloversão, linguoversão, giroversão, perfil facial, posição dos lábios, assimetria facial, respiração e deglutição, mastigação, fonação e cronologia de erupção dos dentes. 


\subsection{Análise Estatística}

Os dados foram tabulados no Microsoft Excel 2010 (Microsoft Windows, Novo México, EUA) e posteriormente submetidos aos testes de Mann-Whitney, Igualdade de Duas Proporções e Qui-Quadrado empregando-se o nível de significância de 5\% (p<0,05).

\section{RESULTADOS}

O gráfico 1 descreve a média de idade (anos) dos indivíduos da amostra e a média de tempo (meses) de amamentação natural e artificial.

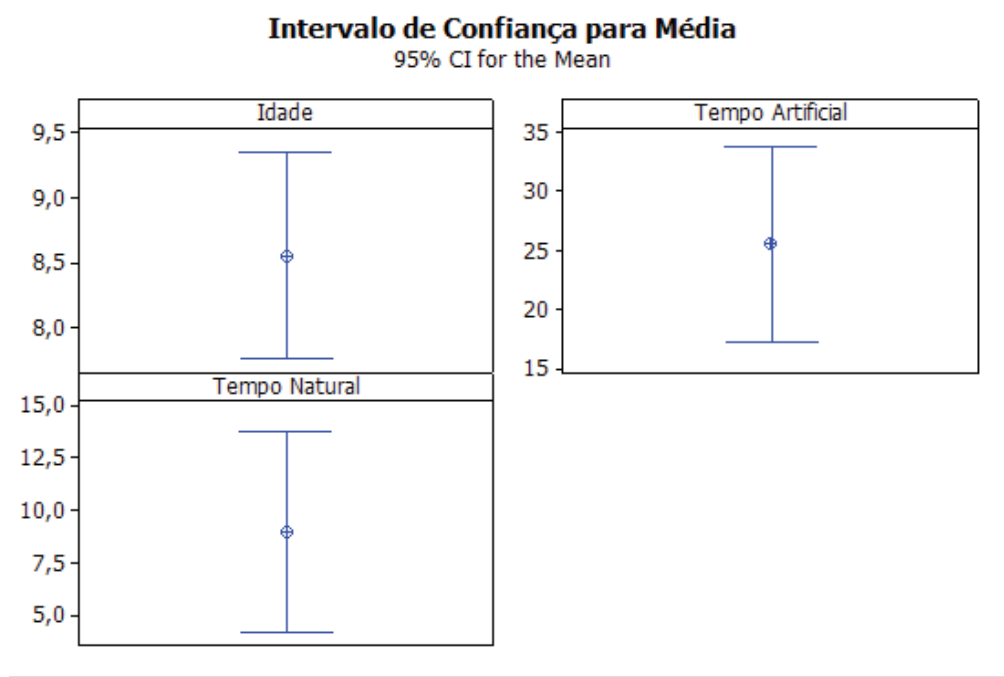

Gráfico 1: Intervalo de Confiança para Média das Variáveis Quantitativas Fonte: Elaboração da autora (2017)

As porcentagens de dentadura decídua, mista e permanente dos indivíduos estão representadas no gráfico 2 .

\section{Distribuição de Dentição}

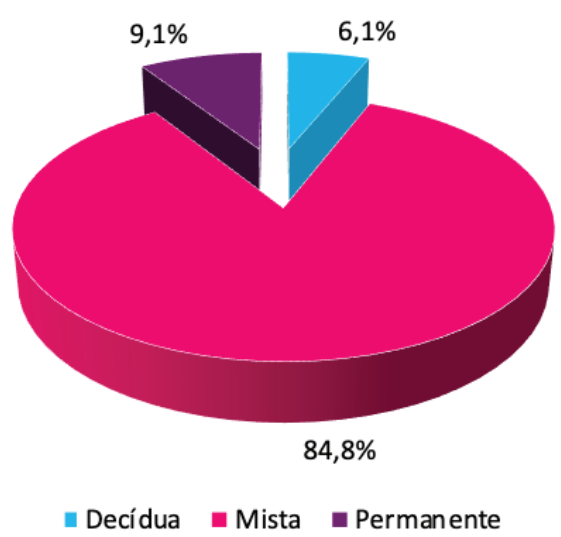

Gráfico 2: Distribuição de Dentição

Fonte: Elaboração da autora (2017) 
O gráfico 3 descreve a distribuição de Classificação de Angle, em que o número de indivíduos de Classe III de 9,7\% foi estatisticamente menor que o número de indivíduos com Classe I e Classe II, embora 54,8\% crianças foram observadas com Classe I e 35,5\% Classe II os quais são numericamente diferentes porém estatisticamente são semelhantes.

\section{Distribuição de Classificação de Angle}

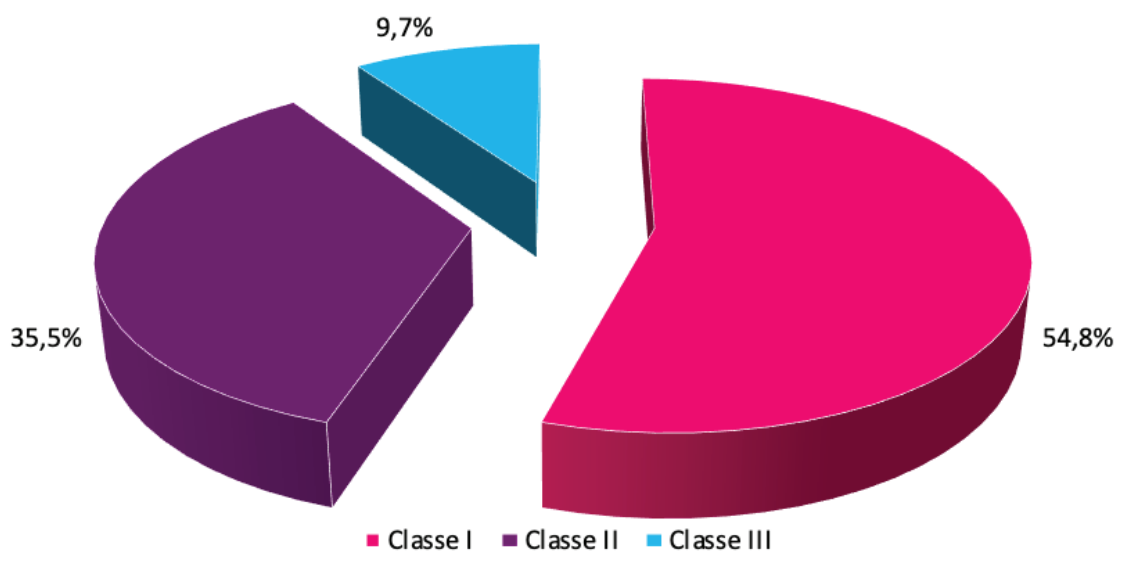

Gráfico 3: Distribuição de Classificação de Angle

Fonte: Elaboração da autora (2017)

A tabela 1 mostra a distribuição da maloclusão.

Tabela 1: Distribuição de maloclusão

\begin{tabular}{l|l|l|l}
\hline Maloclusão & $\mathrm{N}$ & $\%$ & P-valor \\
\hline Não tem & 5 & $15,2 \%$ & \multirow{2}{*}{$<0,001$} \\
\hline Tem & 28 & $84,8 \%$ & \\
\hline Total & 33 & & \\
\hline
\end{tabular}

A tabela 2 mostra a distribuição de mordida aberta.

Tabela 2: Distribuição de mordida aberta

\begin{tabular}{l|l|l|l}
\hline Mordida aberta & $\mathrm{N}$ & $\%$ & $\mathrm{P}$-valor \\
\hline Não tem & 16 & $80,0 \%$ & \multirow{2}{*}{$<0,001$} \\
\hline Tem & 4 & $20,0 \%$ & \\
\hline Total & 20 & & \\
\hline
\end{tabular}

Fonte: Elaboração da autora (2017) 
A tabela 3 mostra a distribuição de Mordida Cruzada.

Tabela 3: Distribuição de Mordida Cruzada

\begin{tabular}{l|l|l|l}
\hline Mordida Cruzada & $\mathrm{N}$ & $\%$ & P-valor \\
\hline Anterior & 4 & $33,3 \%$ & 0,408 \\
\hline Anterior/Posterior & 2 & $16,7 \%$ & 0,083 \\
\hline Posterior & 6 & $50,0 \%$ & Ref. \\
\hline Total & 12 & & \\
\hline
\end{tabular}

Fonte: Elaboração da autora (2017)

A tabela 4 demonstra a distribuição de sobressaliência e sobremordida, onde ambas apresentaram os mesmos valores.

Tabela 4: Distribuição de Sobremordida e Sobressaliência

\begin{tabular}{l|l|l|l}
\hline Sobressaliência Sobremordida & $\mathrm{N}$ & $\%$ & P-valor \\
\hline Acentuada & 8 & $40,0 \%$ & \multirow{2}{*}{0,206} \\
\hline Não acentuada & 12 & $60,0 \%$ & \\
\hline Total & 20 & & \\
\hline
\end{tabular}

Fonte: Elaboração da autora (2017)

O gráfico 4 tem como objetivo mostrar a distribuição de apinhamento.

\section{Distribuição de "Apinhamento"}

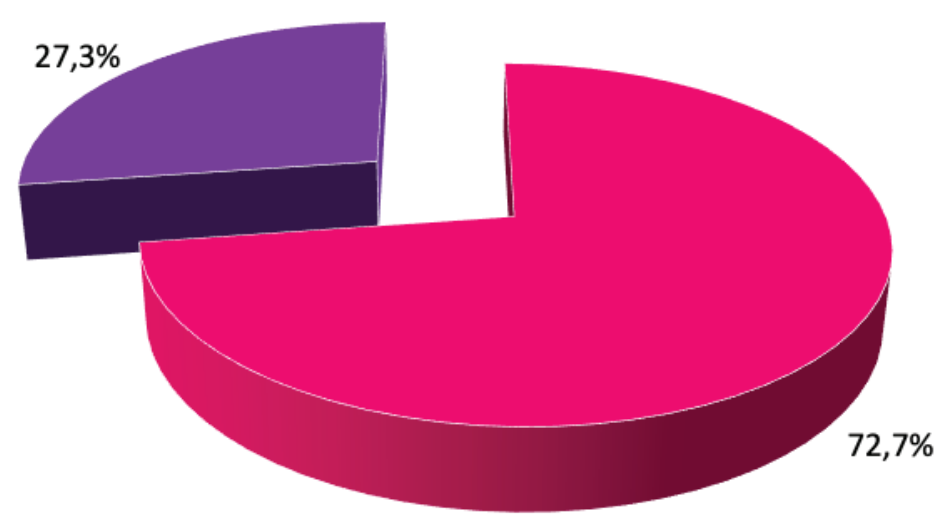

não $\square$ Sim

Gráfico 4: Distribuição de Apinhamento

Fonte: Elaboração da autora (2017) 
A tabela 5 tem como resultado a distribuição de Tipo de Amamentação.

Tabela 5: Distribuição de Tipo de amamentação

\begin{tabular}{l|l|l|l}
\hline Tipo de amamentação & $\mathrm{N}$ & $\%$ & P-valor \\
\hline Artificial & 23 & $69,7 \%$ & \multirow{2}{*}{0,001} \\
\hline Natural & 10 & $30,3 \%$ & \\
\hline Total & 33 & & \\
\hline
\end{tabular}

Fonte: Elaboração da autora (2017)

A tabela 6 mostra a relação do Tipo de Amamentação relacionado com a Maloclusão

Tabela 6: Relação do Tipo de Amamentação com Maloclusão

Fonte: Elaboração da autora (2017).

\begin{tabular}{|c|c|c|c|c|c|c|c|c|}
\hline \multirow{2}{*}{\multicolumn{2}{|c|}{ Tipo de amamentação }} & \multicolumn{2}{|c|}{ Artificial } & \multicolumn{2}{|c|}{ Natural } & \multicolumn{2}{|c|}{ Total } & \multirow{2}{*}{ P-valor } \\
\hline & & $\mathrm{N}$ & $\%$ & $\mathrm{~N}$ & $\%$ & $\mathrm{~N}$ & $\%$ & \\
\hline \multirow{3}{*}{$\begin{array}{l}\text { Classificação de } \\
\text { Angle }\end{array}$} & Classe I & 9 & $43 \%$ & 8 & $80 \%$ & 17 & $55 \%$ & \multirow{3}{*}{0,127} \\
\hline & Classe II & 9 & $43 \%$ & 2 & $20 \%$ & 11 & $35 \%$ & \\
\hline & Classe III & 3 & $14 \%$ & 0 & $0 \%$ & 3 & $10 \%$ & \\
\hline \multirow{3}{*}{ Mordida Cruzada } & Anterior & 3 & $50 \%$ & 1 & $17 \%$ & 4 & $33 \%$ & \multirow{3}{*}{0,435} \\
\hline & Anterior/Posterior & 1 & $17 \%$ & 1 & $17 \%$ & 2 & $17 \%$ & \\
\hline & Posterior & 2 & $33 \%$ & 4 & $67 \%$ & 6 & $50 \%$ & \\
\hline \multirow{2}{*}{ Apinhamento } & Não tem & 4 & $17 \%$ & 6 & $60 \%$ & 10 & $30 \%$ & \multirow{2}{*}{0,014} \\
\hline & Tem & 19 & $83 \%$ & 4 & $40 \%$ & 23 & $70 \%$ & \\
\hline \multirow{2}{*}{ Mordida aberta } & Não tem & 15 & $88 \%$ & 1 & $33 \%$ & 16 & $80 \%$ & \multirow{2}{*}{0,028} \\
\hline & Tem & 2 & $12 \%$ & 2 & $67 \%$ & 4 & $20 \%$ & \\
\hline \multirow{2}{*}{ Sobremordida } & Acentuada & 7 & $41 \%$ & 1 & $33 \%$ & 8 & $40 \%$ & \multirow{2}{*}{0,798} \\
\hline & Não acentuada & 10 & $59 \%$ & 2 & $67 \%$ & 12 & $60 \%$ & \\
\hline \multirow{2}{*}{ Sobressaliência } & Acentuada & 8 & $47 \%$ & 0 & $0 \%$ & 8 & $40 \%$ & \multirow{2}{*}{0,125} \\
\hline & Não acentuada & 9 & $53 \%$ & 3 & $100 \%$ & 12 & $60 \%$ & \\
\hline \multirow{2}{*}{ Maloclusão } & Não tem & 2 & $9 \%$ & 3 & $30 \%$ & 5 & $15 \%$ & \multirow{2}{*}{0,117} \\
\hline & Tem & 21 & $91 \%$ & 7 & $70 \%$ & 28 & $85 \%$ & \\
\hline
\end{tabular}

\section{DISCUSS ÃO}

Os resultados desse estudo permitiram concluir que a $H_{0}$ é verdadeira, pois o tipo ou a duração da amamentação não influenciaram o desenvolvimento de maloclusão.

A média de idade das crianças participantes desse estudo foi de 8,5 anos. Com relação ao tempo de aleitamento materno, verificou-se que 57,7\% das crianças foram amamentadas por um período mínimo de seis meses, sendo a amamentação materna exclusiva ou não. Na amostragem, os hábitos deletérios se mostraram mais prevalentes em crianças do sexo feminino, na faixa etária entre 6 e 9 anos, uma diferença de idade significante comparado a outros autores ${ }^{15-20}$, no qual optou-se por estudar crianças com dentadura decídua completa, em contrapartida nesse estudo a dentadura predominante foi a mista, entrando no segundo período transitório pra mista.

O bebê manifesta a necessidade de sucção desde o momento do nascimento, onde pode ser suprida através da sucção nutritiva ou não nutritiva, sendo que na nutritiva é 
oferecido o alimento necessário para satisfazê-lo, dar proteção e criar laço afetivo com a mãe. Já a sucção não nutritiva não supre essas necessidades. O hábito de sucção pode ser mantido até os 18 ou 24 meses, sendo considerada normal e quando vai além desse período de tempo se torna um hábito ruim porque nessa época a criança já apresenta dentadura decídua completa e então deve começar a mastigação. ${ }^{12}$ Esse estudo mostrou que a maioria das mães amamentou seus filhos naturalmente até os 6 meses, embora nem todas tenha realizado o aleitamento materno exclusivo. A alta prevalência de hábitos na população infantil, segundo a literatura está diretamente associada à duração da amamentação natural. $3-4,7,12-16,21$

Foi observada a prevalência de Classe I de Angle em 54,8\% crianças, devido a amostra ter um número maior de crianças apresentando dentição mista, mas também foram encontradas 35,5\% Classe II e 9,7\% Classe III, correlacionando estudos onde a prevalência de Classe II também foi encontrada com predominância. ${ }^{19,22}$

No estudo foi encontrada a sobressaliência acentuada em $8(40 \%)$ crianças e não acentuada em $12(60 \%)$ e para sobremordida $8(40 \%)$ acentuada e não acentuada em 12 $(60 \%)$, sendo comparadas com um estudo que foram mais comumente observadas a sobremordida moderada e severa $(71,8 \%)$ e sobressaliência moderada e severa $(52,1 \%) .{ }^{20}$ Em contrapartida, a prevalência de sobremordida encontrada nesta pesquisa foi considerada elevada comparada à um artigo cujo resultado foi de $18,75 \% .{ }^{23} \mathrm{O}$ alto índice de de sobressaliência também foi constatado em outras pesquisas com resultado de $50 \% .^{24}$

De 12 crianças que mostraram ter mordida cruzada, a mordida cruzada posterior foi verificada em $6(50,0 \%)$ delas, mordida cruzada anterior/posterior em 2 (16,7\%), mordida cruzada anterior em 4 (33,3\%), valores aproximados ao estudo de um autor cujo obteve 1,9\% $(n=14)$ para a mordida cruzada anterior, $6,54 \%(n=48)$ para a mordida cruzada posterior. ${ }^{20}$

Mordida aberta anterior foi considerada quando as bordas incisais dos incisivos centrais decíduos inferiores se encontram abaixo dos incisivos centrais decíduos superiores criando uma ausência de contato oclusal e prevalência de $72 \%$ de mordida aberta. ${ }^{20}$ Esta foi observada em apenas $20,0 \%$ da amostra, um valor muito inferior ao encontrado em outros artigos. ${ }^{12,17,19,21,25}$ Essa discrepância pode ter ocorrido devido ao número reduzido de indivíduos nessa amostra que embora pequena, foi submetida a exame clinico minucioso, ao passo em que alguns artigos foi feito estudo epidemiológico observacional. ${ }^{14,18-20}$ A tendência a mordida aberta de quem teve amamentação natural possuir maior chance de ter maloclusão do que as que tiveram artificial pode ser explicada ao fato de uma das crianças desse grupo chupar dedo em conjunto com chupeta, outra utilizar mamadeira até 4 anos, sendo que elas apresentaram dentição mista onde os dentes ainda estão erupcionando, sendo possivelmente uma mordida aberta transitória, não sendo considerada mordida aberta e sim um período fisiológico normal.

Em relação ao apinhamento, 72,7\% não apresentaram contra 27,3\% que apresentaram, ou seja, não houve influência significativa na presença de apinhamento dentário relacionado à amamentação, assim comparado a outro estudo cujo obteve um valor de 11,2\% de crianças com apinhamento ${ }^{20}$, pode-se concluir que não existiu relação estatisticamente significante do tipo de amamentação com o apinhamento, são variáveis estatisticamente independentes. 
A amamentação artificial média foi de 2 anos e não impactou na maloclusão porque a maioria das crianças foi amamentada por 6 meses ou mais, sendo a média natural de 10 meses, e pelo fato de as crianças terem dentição predominantemente mista. As crianças que não foram amamentadas de forma exclusiva artificial tiveram menor índice de maloclusões e não foi correlacionado estatisticamente porque receberam artificial por um curto período porém também recebiam a natural e forçava a criança a fazer a sucção como deve e isso faz com que as crianças não desenvolvam outros hábitos. As crianças que receberam aleitamento artificial por um período maior, aproximadamente até os 4 anos de idade, apresentaram hábitos deletérios como apertamento e onicofagia, comparado ao estudo onde a frequência de hábitos de sucção foi mais elevada entre as crianças com alimentação artificial do que nas crianças com alimentação natural. ${ }^{3} \mathrm{~A}$ amamentação em si não torna possível a maloclusão, pois o indivíduo realiza em um curto período de tempo. Ou seja, se o estímulo da amamentação artificial for retirado antes da irrupção dos dentes permanentes, pode ocorrer uma autocorreção natural com equilíbrio oclusal e se permanecer após esse período, pode gerar uma maloclusão. como diz o estudo em que as crianças que foram amamentadas por mais de 12 meses tiveram um risco vinte vezes menor para o desenvolvimento de mordida cruzada posterior em comparação com crianças que nunca foram amamentadas e um risco 5 vezes menor do que as amamentadas entre 6 e 12 meses. ${ }^{4}$

Com relação a maloclusão, 91\% das crianças que tiveram aleitamento artificial apresentaram, sendo comparado ao estudo em que foi considerada importante correlação entre os tipos de maloclusão e os aspectos morfofuncionais, mostrando necessidade de intervenção no tempo apropriado. ${ }^{26}$ e $70 \%$ das que realizaram aleitamento natural, sendo que $85 \%$ da amostra total tinha maloclusão, por ser uma mostra de conveniência. Mas quem recebeu artificial teve maior chance de desenvolver maloclusão, em valores absolutos porem estatisticamente não teve diferença, sendo considerada maloclusão quem apresentou ao menos uma delas, exceto Classe I de Angle.

\section{CONCLUSÃO}

- O tipo de aleitamento não influenciou o desenvolvimento de maloclusão.

- O maior tempo de amamentação não indicará, necessariamente, maior tendência a maloclusão

\section{REFERÊNCIAS}

1. Ferraz, M.C.A. Manual prático de Motricidade Oral: Avaliação e tratamento. 5 Ed. Rio De Janeiro: Revinter, 2001; 5: 141.

2. Adair SM. Pacifier use in children: a review of recent literature. Pediatr Dent. 2003; 25:449-58.

3. Leite-Cavalcanti A, Medeiros-Bezerra PK, Moura C. Aleitamento natural, aleitamento artificial, hábitos de sucção e maloclusões em pré-escolares brasileiros. Rev. Salud Publica. 2007; 9 (2): 194-204.

4. Kobayashi H, Scavone H, Ferreira R, Garib D. Relationship between breastfeeding duration and prevalence of posterior crossbite in the deciduous dentition. American Journal of Orthodontics and Dentofacial Orthopedics. 2010;137(1):54-58. 
5. Albuquerque Junior H, Barros A, Braga J, Carvalho M, Maia M. Hábito bucal deletério e má-oclusão em pacientes da Clínica Infantil do Curso de Odontologia da Universidade de Fortaleza. Revista Brasileira em Promoção da Saúde. 2007;40-45.

6. Boeck E, Pizzol K, Barbosa E, Pires N, Lunardi N. Prevalência de má oclusão em crianças de 3 a 6 anos portadoras de hábito de sucção de dedo e/ou chupeta. Revista de Odontologia da UNESP. 2013; 42(2):110-116.

7. Cruz de Almeida, ME, Silva Melo, N, de Alencar Maia, S, Melo Moutinho da Costa, A, Rebelo de Souza, K. A influência do desmame precoce no desenvolvimento de hábitos bucais deletérios. ConScientiae Saúde [Internet]. 2007;6(2):227-234.

8. Katz, Cíntia Regina Tornisiello; Rosenblatt, Aronita; Gondim, Pedro Paulo Costa. Hábitos de sucção, padrão de crescimento facial e alterações oclusais dentárias em pré- escolares do Recife - PE. Bras Ortodon Ortop Facial. Curitiba. 2002; 40 (7): 306-313.

9. Santos Shirley A. dos, Holanda Ana Larissa F. de, Sena Marina F. de, Gondim Líbia A. M., Ferreira Maria Ângela F. Hábitos de sucção não nutritiva em crianças pré-escolares. J. Pediatr. (Rio J.) [Internet]. 2009; 85(5): 408-414.

10. Bresolin, A.M.B.; Lima, I.N.; Penna, H.A.O. Et Al. Alimentação Da Criança. In: Marcondes, E; Vaz, F.A.C.; Ramos, J.L.A. et al. Pediatria Básica: tomo I pediatria geral e neonatal. São Paulo: Sarvier, 2003; 9: 61-96.

11. Cavassani Valdinês G.S., Ribeiro Sonize G., Nemr Nair K., Greco Ana M., Köhle Juliana, Lehn Carlos N.. Hábitos orais de sucção: estudo piloto em população de baixa renda. Rev. Bras. Otorrinolaringol. [Internet]. 2003 Jan [cited 2017 Oct 30]; 69(1): 106-110.

12. Albuquerque S, Duarte R, Cavalcanti A, Beltrão É. A influência do padrão de aleitamento no desenvolvimento de hábitos de sucção não nutritivos na primeira infância. Ciência \& Saúde Coletiva. 2010;15(2):371-378.

13. Suga SS, Bonecker MJS, Sant'anna GR, Duarte DA. Caderno de odontopediatria: ortodontia na dentadura decídua - diagnóstico, planejamento e controle. São Paulo: Santos, 200: 62.

14. Bastos De Oliveira, A, Pereira De Souza, F, Magalhães Leal Chiappetta, Ald. Relação entre hábitos de sucção não-nutritiva, tipo de aleitamento e má oclusões em crianças com dentição decídua. Revista Cefac [Internet]. 2006;8(3):352-359.

15. Ferreira FV, Marchionatti AM, Oliveira MDM, Praetzel JR. Associação Entre A Duração Do Aleitamento Materno E Sua Influência Sobre O Desenvolvimento De Hábitos Orais Deletérios. Rev Sul-Bras Odontol. 2010 Mar;7(1):35-40.

16. Morais Suelly Pinto Teixeira De, Mota Eduardo Luiz Andrade, Amorim Leila Denise A. F.. Fatores Associados À Incidência De Maloclusão Na Dentição Decídua Em Crianças De Uma Coorte Hospitalar Pública Do Nordeste Brasileiro. Rev. Bras. Saude Mater. Infant. [Internet]. 2014 Dez [Citado 2017 Out 16]; 14(4): 371-382. 17.

17. Medeiros Bezerra, PK, Leite Cavalcanti, A, Medeiros Bezerra, P, Moura, C. Maloclusões, Tipos de aleitamento e hábitos bucais deletérios em Pré-Escolares - Um estudo de associação. Pesquisa Brasileira em Odontopediatria e Clínica Integrada [Internet]. 2005;5(3):267-274.

18. Degan VV, Puppin-Rontani RM. Prevalence Of Pacifer-Sucking Habits And Successful Methods To Eliminate Them- A Preliminary Study. J Dent Child (Chic). 2004; 71(2): 148-51.

19. Lima GN, Cordeiro CM, Justo JSJ, Rodrigues LCB. Mordida Aberta Anterior E Hábitos Orais Em Crianças. Rev Soc Bras Fonoaudiol. 2010;15(3):369-75.

20. Mendes ACR, Valenca AMG, Lima CCM. Associacao Entre Aleitamento, Habitos De Succao Nao-Nutritivos E Ma Oclusoes Em Criancas De 3 A 5 Anos. Cienc Odontol Bras. 2008; 11(1): 67-75.

21. Heringer MRC, Reis M, Pereira LFS, Di Ninno CQMS. A Influência Da Amamentação Natural No Desenvolvimento Dos Hábitos Orais. Rev CEFAC. 2005; 7 (3): 307- 10.

22. Pizzol, K. E. D. C.; Montanha, S. S.; Fazan, E. T.; Boeck, E. M.; Rastelli, A. N. S. Prevalência dos hábitos de sucção não nutritiva e sua relação com a idade, gênero e tipo de aleitamento em Pré-Escolares da cidade de Araraquara. Rev CEFAC, 2012;14,(3): 506- 515.

23. Da Silva R, Jóias R, Josgrilberg E, Rode S, Paranhos L, Joias R. The correlation between malocclusions and morphofunctional aspects: analysis of patients aged from 7 to 12 years old. Brazilian Dental Science. 2016;19(4):90.

24. Thomaz EBAF. Relação Entre O Estado Nutricional e Má-Oclusão Em Pré-Escolares Na Cidade De São Luís (MA) [Dissertação]. João Pessoa: Faculdade De Odontologia, Universidade Federal Da Paraíba; 2005;12(2):212-24.

25. Tollara MCRN, Duarte DA, Bönecker M, Pinto VG. Estudo Epidemiológico Da Prevalência De Maloclusão Em Crianças De 5 A 35 Meses De Idade. Rev Assoc Paul Cir Dent. 2003 Jul/Ago; 57 (4):267-73.

26. Silva, Regiane \& Jóias, Renato \& Josgrilberg, Erika \& Rode, Sigmar \& Paranhos, Luiz \& Jóias, Renata. (2016). The correlation between malocclusions and morphofunctional aspects: analysis of patients aged from 7 to 12 years old. Brazilian Dental Science. 19. 90. 10.14295/bds.2016.v19i4.1304. 